\title{
Search for the Quark-Gluon Plasma (1989): \\ The NA35 Experiment at the CERN SPS
}

H.G. Pugh

LBL --27833

DE90 002070

\author{
Nuclear Science Division \\ Lawrence Berkeley Laboratory \\ 1 Cyclotron Road \\ Berkeley, CA 94720
}

and

The NA35 Collaboration

J. Bartke, ${ }^{5)}$ H. Bialkowska, ${ }^{12)}$ R. Bock, ${ }^{6)}$ R. Brockmann, ${ }^{6)}$ S.I. Chase, ${ }^{3)}$

C. De Marzo, ${ }^{2)}$ I. Derado, ${ }^{10)}$ V. Eckardt, ${ }^{10)}$ J. Eschke, ${ }^{7)}$ C. Favuzzi, $\left.{ }^{2}\right)$

J. Fent, ${ }^{10)}$ D. Ferenc, ${ }^{13)}$ H. Fessler, ${ }^{(0)}$ P. Freund, ${ }^{(0)}$ M. Gazdzicki, ${ }^{(1)}$

H.J. Gebauer, ${ }^{10)}$ E. Gladysz, ${ }^{4)}$ C. Guerra, ${ }^{6)}$ J.W. Harris, ${ }^{3)}$ E. Hartig, ${ }^{7)}$

W. Heck, ${ }^{7)}$ T. Humanic, ${ }^{6}$ K. Kadija, ${ }^{13)}$ A. Kühmichel, ${ }^{7)}$ M. Lahanas, ${ }^{7}$

Y. Lee, ${ }^{7)}$ S. Margetis, ${ }^{7)}$ E. Nappi, ${ }^{2)}$ G. Odyniec, ${ }^{3)}$ G. Paic, ${ }^{13)}$

A.D. Panagiotou, ${ }^{1,4)}$ A. Petridis, ${ }^{1)}$ J. Pfennig, ${ }^{7)}$ F. Posa, ${ }^{2)}$ K.P. Pretzl, ${ }^{10)}$

H.G. Pugh, ${ }^{3)}$ F. Pühlhofer, ${ }^{9)}$ G. Rai, ${ }^{3)}$ A. Ranieri, ${ }^{2)}$ W. Rauch, ${ }^{3)}$

R. Renfordt, ${ }^{7}$ D. Röhrich, ${ }^{7}$ H. Rothard, ${ }^{7)}$ T. Schouten, ${ }^{10)}$ L.S. Schroeder, ${ }^{3)}$

G. Selvaggi, ${ }^{2)}$ P. Seyboth, ${ }^{10)}$ J. Seyerlein, ${ }^{10)}$ E. Skrzypzcak, ${ }^{11)}$ P. Spinelli, ${ }^{2)}$

R. Stock, ${ }^{4,7)}$ H. Ströbele, ${ }^{7)}$ A. Thomas, ${ }^{7)}$ L. Teitelbaum, ${ }^{3)}$ S.R. Tonse ${ }^{3)}$

G. Vesztergombi, ${ }^{10)}$ D. Vranic, ${ }^{13)}$ S. Wenig, ${ }^{7)}$ and M. Wensveen ${ }^{6)}$

\begin{abstract}
Results from the NA35 experiment at the CERN SPS are described in the context of possible formation and identification of a quark-gluon plasma (QGP). Evidence is presented that the initial energy density and temperature are sufficient for the QGP to be produced, and that hydrodynamic flow occurs in the exparision stage. Evidence for an unexpectedly large pion source size and for enhanced strangeness production is presented, and discussed in terms of QGP formation. Plans for experiments in 1990-91 with an expanded set up are presented, and prospects for a program with $\mathrm{Pb}$ beams at the SPS are discussed.
\end{abstract}

1) Physics Dept. Univ. of Athens, 2) Dipartimento di Fisica, Univ. di Bari, 3) Lawrence Berkeley Lab., 4) CERX, 5) Inst. of Nuclear Physics, Cracow, 6) GSI Darmstadu, 7) Fachbereich Physik, Un:v. Frankfurt, 8) Fakultät für Physik, Lniv. Freiburg, 9) Fachbereich Physik, Univ. Marburg, 10) Max-Planck-Institut für Physik, Munchen, 11) Inst. of Experimental Physics, Lniv. of Warsaw. 12) Institute of Nuclear Studies, Warsaw, 13) Rudjer Boskovic Inst. Zagreb. 


\section{Introduction}

Collisions of nuclei at very high energy should create a finite volume of hadronic matter at high energy density. Results from strong interaction lattice gauge theory [1] suggest that above a critical temperature in the region of $200 \mathrm{MeV}$ the hadronic state of matter is iransformed to a new phase consisting of deconfined quarks and gluons with an energy density of a few $\mathrm{GeV} / \mathrm{fm}^{3}$, to be compared with that of ground state nuclear matter which is $150 \mathrm{MeV} / \mathrm{fm}^{3}$. Such extreme conditions could be reached in central nucleus-nucleus collisions by converting the initial relative c.m. energy into intemal excilation of a "fireball" formed at rapidities intermediate between those of target and projectile, provided sufficient beam energy and adequate stopping power.

A pilot program to investigate the properties of such nucleus-nucleus collisions was begun at CERN in 1986, with ${ }^{16} \mathrm{O}$ beams at $60 \mathrm{GeV} /$ mucleon and $200 \mathrm{GeV} /$ nucleon. In 1987 a second run was carried out with ${ }^{32} \mathrm{~S}$ beams at $200 \mathrm{GeV} /$ nucleon. Despite expectations that these runs would provide mainly a first look at the phenomenology of high energy nucleus-nucleus collisions and a great deal of useful experience, both theoretical and experimental, that would be of value in a later phase with heavier projectiles and/or higher beam energies a number of surprises have emerged, including the possible observation of a number of quark-gluon plasma signatures.

\section{Experimental Set-Up}

Figure 1 shows the NA35 experimental set-up. The main components are [2]:

(a) A large-volume Streamer Chamber, $2 \times 1.2 \times 0.7 \mathrm{~m}^{3}$, in a $1.5 \mathrm{~T}$ superconducting vertex magnet. Targets were mounted directly in front of the chamber. Two principal readout systems were employed:

A set of 3 film cameras with Zeiss optics recorded images on high-sensitivity $70 \mathrm{~mm}$ film. They were equipped with two-stage magnetic-focusing ITT image intensifiers of about 250 -fold gain. The spatial resolution was of the order of $2 \mathrm{~mm}$. The film is analyzed off line in Berkeley, 
Cracow, Frankfurt, Marburg, München, Warsaw, and Zagreb. A typical central ${ }^{16} \mathrm{O}+\mathrm{Pb}$ event is shown in Fig. 2.

(b) A set of 3 CCD digitizing cameras, with TI $1024 \times 1024$ byte arrays and equipped with two stages of image intensifiers, recorded the events on line. With the help of a micro-VAX based image processing and display system, events were monitored, and the multiplicity of charged tracks obtained on line [3].

(c) A set of electromagnetic and hadronic calorimeters. The angular domain $\theta<0.3^{\circ}$ (nuclear projectile fragmentation) is covered by a 4-segment "veto" calorimeter. The subsequent interval, $0.3^{\circ}-2.2^{\circ}$, has a continuous single-cell electromagnetic and hadronic calorimeter. The larger angle domain is covered in high granularity by a Photon Position Detector (PPD) consisting of altemating layers of lead and planes of proportional tubes read out by 3072 ADC channels, and by a Ring Calorimeter divided into 240 cells ( 24 in azimuth and 10 in radius with sizes chosen to cover equal units of rapidity at $200 \mathrm{GeV} /$ nucleon).

This set of calorimeters is movable: at $200 \mathrm{GeV} /$ nucleon it covered a $2.3^{\circ}-12.5^{\circ}$ angular range corresponding to $2.0<\mathrm{X}<3.8$ and at $60 \mathrm{GeV} /$ nucleon it covered a $4.3^{\circ}-20.5^{\circ}$ range corresponding to $1.7<y<3.3$. Some measurements were also made at other settings.

Two principal trigger modes were employed. From the Veto Calorimeter various levels of projectile energy degradation could be selected, ranging from, "minimum bias" to "central collision," the later requiring typically $\mathrm{E}_{\text {veto }}<0.1 \mathrm{E}_{\mathrm{beam}}$. The other trigger was observed from the transverse energy of the produced photons (mainly from $\pi^{\circ}$ decay), recorded in the PFD. On the average 3 Steamer Chamber events were recorded per extraction spill, and in addition about 100 events in the calorimeters without accompanying streamer chamber information. A total of more than 100,000 Streamer Chamber and 2,500,000 calorimeter events have been recorded, for beams of ${ }^{16} \mathrm{O}$ at $60 \mathrm{GeV} /$ nucleon and $200 \mathrm{GeV} /$ nucleon. A corresponding volume of data was recorded for ${ }^{32} \mathrm{~S}$ beams at $200 \mathrm{GeV} /$ nucleon. In addition data were taken for $60 \mathrm{GcV}$ and $200 \mathrm{GeV}$ 
incident protons.

Analysis of the Streamer Chamber data is a difficult, slow, and ongoing process. Results for $60 \mathrm{GeV} /$ nucleon and $200 \mathrm{GeV} /$ hucleon ${ }^{16} \mathrm{O}$ beams have been published on energy flow [4], cross sections and multiplicity distributions [5], two-pion correlations [6.7], rapidity and transverse momentum distributions [8], and strangeness production [9]. Some preliminary results for $200 \mathrm{GeV} /$ nucleon ${ }^{32} \mathrm{~S}$ beams have also been presented [10]. Reviews of the experiment can be found in refs. [11-13]. In this report the published results are surveyed briefly and some new, preliminary, results are presented to indicate present lines of research and interpretation. Special emphasis is given to study of the ${ }^{32} \mathrm{~S}+\mathrm{S}$ system which presents some special advantages.

\section{General Properties of the Reaction}

Here we focus on properties which are directly relevant to the question of whether the conditions produced in the interaction are those in which a quark-gluon plasma might be formed [14]. Transverse energy spectra are discussed for their relevance to the energy density produced in the collision. The transverse momentum distributions of particles are examined for evidence on whether the temperatures produced are sufficient for quark-gluon plasma production. Finally, evidence for hydrodynamic flow is reviewed.

\subsection{Energy Density}

The energy density in the interaction region at time $t$ after the collision is given by Bjorken [15] as

$$
\varepsilon=\frac{1}{\pi\left(r_{0} A^{1 / 2}\right)^{2}} \cdot \frac{1}{t} \frac{d E_{T}}{d y}
$$

The energy is taken as the transverse energy $\mathrm{dE}_{\mathrm{T}}$ within a rapidity interval dy. The first term in the expression is the cross sectional area of the interaction zone. The term tdy is the length of the element dy at time $t$ after the collision. 
The quantity $\mathrm{dE}_{\mathrm{T}} / \mathrm{dy}$ can be measured by calorimetry since the NA35 calorimeters are divided radially and the measured energy in each element can be projected on to the transverse direction. Furthermore there is a good correspondence near mid-rapidity between angle and rapidity.

Figure 3 shows a transverse energy spectrum obtained for the ${ }^{16} \mathrm{O}+\mathrm{Pb}$ system at 200 GeV/nucleon. This was the first result obtained from the NA35 experiment in 1986 [4]. A broad distribution is due to variation of the ${ }^{16} \mathrm{O}+\mathrm{Pb}$ impact parameter and the shoulder at $80 \mathrm{GeV}$ corresponds to central collisions in which all the nucleons in the ${ }^{16} \mathrm{O}$ projectile interact with the target. These are the collisions of most interest.

For central collisions we can estimate from these results within the acceptance $2.2<y<3.8$ that $\mathrm{dE}_{\mathrm{T}} / \mathrm{dy} \approx 50 \mathrm{GeV}$, and for $\mathrm{t}=1 \mathrm{fm} / \mathrm{c}$ the energy density $\varepsilon \approx 1.8 \mathrm{GeV} / \mathrm{fm}^{3}$, with fluctuations up to about $2.5 \mathrm{GeV} / \mathrm{fm}^{3}$. Similar measurements for ${ }^{32} \mathrm{~S}+$ Au yield values of $\varepsilon$ about $20 \%$ greater.

We may conclude from these results that there is an a priori case that the energy density of $2 \mathrm{GeV} / \mathrm{fm}^{3}$ estimated to be necessary for quark-gluon plasma formation may have been reached. This estimate of $\varepsilon$ is, unfortunately, very imprecise for two important reasons. One is that the choice of $t=1 \mathrm{~m} / \mathrm{c}$ is somewhat arbitrary. It is chosen to correspond to the hadronization time $\tau_{\mathrm{o}}$, the time required to form a hadron. This quantity is not well understood. The other is that the formula is derived under assumptions appropriate at infinite energies. In particular the formation time of the hot system is assumed to be zero, and the system after formation is assumed to be a thin disk. This is at variance with the actual situation at $200 \mathrm{GeV} /$ nucleon, where the transition time for an ${ }^{16} \mathrm{O}$ projectile to pass through a Pb nucleus is approximately $2 \mathrm{fm} / \mathrm{c}$.

An interesting result that emerged from this first measurement was that the $E_{T}$ 
distribution for central collisions could be well explained as a convolution of 16 independent $p+$ $\mathrm{Pb} \mathrm{E}_{\mathrm{T}}$ distributions (actually $\mathrm{p}+\mathrm{Au}$ v/as used, but the result should not be very sensitive to the target mass). This is shown as the solid curve in Figure 3.

Similar convolutions directly relating central ${ }^{16} \mathrm{O}$-nucleus collisions to p-nucleus collisions have been successful (at various levels of accuracy) also for multiplicities (5), strangeness production (9), and $\mathrm{p}_{\mathrm{T}}$ distributions (8). It is not found that a convolution of $\mathrm{p}-\mathrm{p}$ collisions describes p-nucleus collisions in a simple way. The success of the p-nucleus to central ${ }^{16} \mathrm{O}$-nucleus convolution hinges on whether the 16 nucleons in ${ }^{16} \mathrm{O}$ interact independently with the target. ${ }^{16} \mathrm{O}$ is rather a dilute nucleus, but some additional help is needed. It may be that nucleon-nucleon collisions contributing significantly to large transfers of transverse momentum or energy are better localized than the average, so that for some processes the nucleons in the ${ }^{16} \mathrm{O}$ nucleus appear more point-like than for others.

\subsection{Temperature}

The next question is whether sufficient temperature is reached in the interaction volume to create a quark gluon plasma. This question is not easy to address because any equilibrium reached is at best transient, and because the system is expected to expand and cool down before emitting the particles which are observed. The expansion may also be complicated by hydrodynamic flow, of interest in itself.

Relativistic thermodynamics has been developed extensively by Hagedorn [16]. Here we refer to some of the simpler results, which are worth laying out in detail because some confusion exists in the literature.

For a single (isotropic) firebal!, the momentum distribution for emitted particles is given in the c.m. by:

$$
\frac{d n}{d p^{3}}=\text { const. } \frac{1}{e^{F / T} \pm d}
$$


where $d=+1,-1,0$ for a Fermi, Bose, or Boltzmann distribution, $E$ is the total energy of the particle and $\mathrm{T}$ is the temperature. After integrating over the azimuthal angle, this becomes [8]:

$$
\frac{d n}{d y d p_{T}}=\text { const. } \frac{p_{T} m_{T} \operatorname{coshy}}{e^{m_{T} \operatorname{coshy} / T_{ \pm d}}}
$$

or, using the relationship $\frac{1}{\mathrm{p}_{\mathrm{T}}} \frac{\mathrm{dn}}{\mathrm{dp_{ \textrm {T } }}}=\frac{1}{\mathrm{~m}_{\mathrm{T}}} \frac{\mathrm{dn}}{\mathrm{m}_{\mathrm{T}}}$ :

$$
\frac{d n}{d y d m_{T}}=\text { const. } \frac{m_{T}^{2} \operatorname{coshy}}{e^{m_{T} \operatorname{coshy} y T} \pm d}
$$

At mid-rapidity $(y=0)$ this becomes:

$$
-\frac{d n}{\text { dydm }_{T}}(y=0)=\text { const. } \frac{m_{T}^{2}}{e^{m \tau r} \pm d}
$$

Comparison of this formula with the rapidity distribution $\mathrm{dn} / \mathrm{dy}[8]$ shows that the distribution is too wide to be accounted for by a single fireball, for ${ }^{16} \mathrm{O}+\mathrm{Au}$ at $60 \mathrm{GeV} /$ nucleon and 200 $\mathrm{GeV} /$ nucleon. This situation is familiar from the study of hadron-hadron collision where Hagedom introduced [16] an extra function to allow for the longitudinal dynamics. To investigate the effects of this we have explored the effects of a Gaussian distribution in rapidity for fireballs all with the same temperature:

$$
\frac{d n}{d y_{0}}=\frac{1}{\sigma \sqrt{2} \pi} e^{-\frac{1}{2}\left(\frac{y_{0}-y_{m}}{\sigma}\right)^{2}}
$$

We find that the data require $\sigma \approx 1.0$. A global fit can then be attempted using the expression:

$$
\frac{d n}{d y d m_{T}}=\text { const. } \int_{-\infty}^{\infty} \frac{1}{\sigma \sqrt{2} \pi} e^{-\frac{1}{2}\left(\frac{y_{0}-y_{\text {deam }}}{\sigma}\right)^{2}} \cdot \frac{m_{T}^{2} \cosh \left(y_{0}-y\right)}{e^{m \pi \cosh \left(y_{0}-y\right) / T} \pm d} d y_{0}
$$

This work is in progress.

For preliminary investigations we have noted that for a Boltzmann distribution equation (4) takes on a specially simple form; 


$$
\frac{d n}{d m_{T}}(y=0)=\text { const. } m_{\mathrm{T}}^{2} \mathrm{e}^{-\mathrm{m}_{\mathrm{T}} / \mathrm{T}}
$$

Equations (3) and (6) also become (for a Boltzmann distribution) specially simple after integrating over rapidity

$$
\frac{\mathrm{dn}}{\mathrm{dm}_{\mathrm{T}}}=\text { const } \mathrm{m}_{\mathrm{T}}^{2} \mathrm{~K}_{1}\left(\mathrm{~m}_{\mathrm{T}} / \mathrm{T}\right)
$$

where $\mathrm{K}_{1}\left(\mathrm{~m}_{\mathrm{T}} / \mathrm{T}\right)$ is a modified Bessel funct:on, and

$$
\frac{\mathrm{dn}}{\mathrm{dm}_{\mathrm{T}}} \rightarrow \text { const } \mathrm{m}_{\mathrm{T}}^{3 / 2} \mathrm{e}^{-\mathrm{m}_{\mathrm{T}} / \mathrm{T}} \text { for large } \mathrm{m}_{\mathrm{T}} / \mathrm{T}
$$

Near $y=0, \frac{d n}{d n_{T}}$ for distribution (6) is closer to ec 9,9 than to eq. 7 . We have therefore examined whether $m_{\mathrm{T}}^{-3 / 2} \frac{\mathrm{dn}}{\mathrm{dm}_{\mathrm{T}}}$ is an exponentally decaying function of $\mathrm{m}_{\mathrm{T}} / \mathrm{T}$ at large $\mathrm{m}_{\mathrm{T}}$.

Figures 4,5 and 6 show plots of this type for a variety of particles, in the reactions ${ }^{16} \mathrm{O}+$ $\mathrm{Au},{ }^{32} \mathrm{~S}+\mathrm{S}$, and $\mathrm{p}+\mathrm{Au}$ at $200 \mathrm{GeV} /$ nucleon. It is remarkable that in every case except for pions at lnw $m_{\mathrm{T}}$ the data are qualitatively approximated by a straight line fit with a temperature of $\mathrm{T}$ $200 \mathrm{MeV}$, similar to that theoretically predicted for formation of a quark gluon plasma. A fit using eq. (7) instead of eq. (9) would give a slightly lower temperature.

The conclusion that $\mathrm{T}=200 \mathrm{MeV}$ is preliminary, and awaits a more careful analysis. It is, however, based on a consistently defined thermal model, and is thus distinguished from some other fits that have been reported in the literature. In a report of our work on strangeness production [9], we used the relationship

$$
\frac{\mathrm{dn}}{\mathrm{dm}} \rightarrow \text { const. } \mathrm{m}_{\mathrm{T}} \mathrm{e}^{\mathrm{m}_{\mathrm{T}} \mathrm{T}_{\mathrm{er}}}
$$

This was also used in Reference [17]. Equation 10 arises from the assumption that

$$
E \frac{d \sigma}{d p^{3}}=\text { const } e^{-m_{T} / T_{\text {er }}}
$$


which corresponds to different dynamics from that of eq. 2 . Unfortunately, it is usually possible tc fit a variety of different exponential forms to the data. Each leads to a different valuc of extracted "temperature." The use of eq. (10) instead of eq. (\$) lowers the extracted temperature by a factor $=\left(1+\frac{T}{2 m_{T}}\right)$. Fitting the entire spectram may help resolve these differences.

\subsection{Expansion and Hydrodynamical Flow}

The preceding discussion of spectra in terms of a thermal distribution is an oversimplification. Complicating factors occur, including the decay of resonances, expansion and cooling prior to the emission of particles, and perhaps hydrodynamic flow. It is onj" at high transverse momenta that the effects of initial temperature may survive, as suggested in our data.

Among these effects, hydrodynamic flow is specially interesting, since if identified it could give an indication of the equation of state of the high-density nuclear matter in the interaction zone. For orientation, we note that the velocity of sound in an ultrarelativistic gas is $\mathrm{c} / \sqrt{3}$. From this we can estimate that the effects of hydrodynamic flow would be felt in the region $\mathrm{m}_{\mathrm{T}} \leq 1.22 \mathrm{~m}$, or $\mathrm{p}_{\mathrm{T}} \leq 0.7 \mathrm{~m}$, The comparison of spectra for different mass particles was suggested by Siemens and Rasmussen [18] as a way to identify flow.

Lee and Heinz [19] have recenty developed a model incorporating most of these complicating features, and have obtained good fits to $\pi^{\circ}$ spectra from WA80 and to $\pi^{-}$spectra from NA35. The radial flow velocicy extracted averages $-c / 2$, the freeze-out temperarure is about $100 \mathrm{MeV}$, and the initial temperature before expansion is about $200 \mathrm{MeV}$, consistent with the tentative conclusion we drew from data of figs. 4-6.

In order to confirm the conclusions of Lee and Heinz, we used their formalism to analyze simultaneously spectra of $\pi^{-}, \mathrm{K}^{\circ}, \Lambda^{\circ}$ and $\bar{\Lambda}^{\circ}$ measured in the reaction $\mathrm{O}+\mathrm{Au}$ at $200 \mathrm{GeV} /$ nuclcon [13]. The same set of parameters, only slightly adjusted from reference 19, fits all the data 
adequately, confirming the existence of flow, previously indicated only by the concave shape of the $\pi^{\circ}$ and $\pi^{-}$spectra. The fits are shown in Figure 7.

While the model of Lee and Hein $<$ permits the extraction of rather precise values for the initial temperature and for the flow velocity, it is not sensitive to the initial energy density. For this, independent measurements of, or assumptions concerning, the volume of the initial system are necessary, just as in use of the Bjorken formula, eq. (1). This must, at the present time, be regarded as an unsolved problem. Measurements of freeze-out radii by means of two-pion correlations will be discussed below.

\section{A. Some Surprises}

The conclusion of the previous sections, that the initial conditions reached in the collisions are suitable for production of a quark-gluon plasma, is not a surprise: that was the initial motivation for carrying ouc the studies. However, observation of signatures for production of the plasma wuuld be a surprise, since it was expected that the size of the interaction volume for light projecdiles such as ${ }^{16} \mathrm{O}$ would be too small for equilibrium to be established.

The most striking surprise in the CERN heavy ion program was obtained in the NA38 experiment in which $\mathrm{J} / \Psi$ producuon was observed [20] to have all the properties previously predicted [21] for quark-gluon plasma formation. Various post hoc explanations have been proposed to avoid the necessity of such a conclusion.

In the NA35 experiment two major surprises have been encountered: observation of a large-radius pion source at mid-rapidity, and observation of a substantial enhancement of strange particle production in central ${ }^{32} \mathrm{~S}+\mathrm{S}$ collisions. The latter, especially, finds its most natural explanation in terms of quark-gluon plasma formation. 


\subsection{Oiservation of Pion Source Sizes}

All observed particles are measured only in momentum space, and any information on source sizes is a result of theoretical interpl etation of the spectra. One type of observation is, however, believed to give information on source size in a relatively direct and model-independent way. This is the study of Bose-Einstein correlations between pairs of like particles, in uur experiment negative pions. The simplest analysis assumes a totally chaotic source. An enhancement of the two pion correlation by a factor of two should then appear at zero relative momentum of the pair, the width of the correlation being the Fourier transform of the radial distribution of the source. When this does not work (the enhancement usually being less than a factor of two) a chaoticity parameter $\lambda$ is introduced to represent the fraction of the source which is chaotic. The justification for introduction of inis parameter is not well established.

Further complications arise at very high energies from the extreme relativistic dynamics of the source, in which the strong correlation between position and velocity means that pairs of particles originating in widely different parts of the source may not be able to exhibit Bose-Einstein enhancement because their momenta are too different. For a discussion of these questions in the context of NA35 data, see reference [6]. Further examination has been carried out in particular in refs $[22,23]$.

Correlations between paiss of negative pions in NA35 were presented for central ${ }^{16} \mathrm{O}+\mathrm{Au}$ collisions at $200 \mathrm{GeV} /$ nucleon in [6]. Here are summarized the main results. Near mid-rapidity, a source radius of $8.1 \pm 1.6 \mathrm{fm}$ was observed, with a chaoticity parameter $\lambda=0.77 \pm 0.19$. A way from mid-rapidity a smaller source radius $\sim 4 \mathrm{fm}$ was observed, with a chaoticity parameter $\lambda \sim 0.3$. The radius observed away from mid-rapidity is roughly consistent with the size of the projectile nucleus, though the $\lambda$ parameter is not explained. The chaoticity parameter at midrapidity is consistent with a themal source, whose radius is found to be very large. 
A qualitative explanation of the large radius was proposed in [6]. The large number of pions produced in the reaction is such that as the source expands a radius of the order of $8 \mathrm{fm}$ has to be reached before the pions can be considered to be non-interacting. This defines the freezeout radius which is observed in the correlation experiments. Such a model is testable by studying different interacting systems because the simple model proposed predicts that the freeze-out radius should depend on $\sqrt{\frac{d n}{d v}}$. This can be tested by studying different nuclear systems cr different bombarding energies.

The large source radius does not provide direct evidence of quark-gluon plasma formaiion. If however the radius of $8 \mathrm{fm}$ is a freeze-out radius as interpreted above, it means that at radii of $4 \mathrm{fm}$, corresponding to the presumed initial volume of the interaction region, the particles in the system cannot be pions [24]. It is just not possible to fit them all in. Whether the initial system is better described in terms of a hadronic gas or as a quark-gluon plasma is an open question. It may in the end tum out to be primarily a matter of simplicity and economy of parameters, in which case a description in terms of quarks and gluons will have an advantage.

The measurements of ref [6] involved a considerable increase in precision over previous high-energy studies of such systems as $\mathrm{e}^{+} \mathrm{e}^{-}, \mathrm{p}-\mathrm{p}$ and $\pi$-p. In these, radius parameteĩs $\sim 1 \mathrm{fm}$ are typical, and chaoticity parameters $\lambda-0.5$ are usually found. An eight-fold increase in the radius implies the necessity for an eight-fold improvement in exp srimental momentum resolution and in the ability to resolve close tracks. Also, as the radius increases, the number of pairs close enough in momentum to fall within the range of potential Bose-Einstein enhancement diminishes, while the total number of pairs remains the same.

The measurements of ref [6] were possible only because of the high precision of the sreamer chamber, and becation of the very large number of pion pairs per event (sufficient to extract radius information even from individual events [7]). In our furher studies we have concentrated on a) improving statistics for ${ }^{16} \mathrm{O}+\mathrm{Au}$; b) studying other systems to test the $\sqrt{\frac{\mathrm{dn}}{\mathrm{dy}}}$ dependence 
of source radius; c) refining the momentum resolution and two-track resolution to study better the shape of the correlation.

One reason for studying the shape of the correlation is to try to distinguish the possible effects of resonance production on the results. These effects have been studied in hadron-hadron collisions, though a major difficulty is finding a suitable model for particle production which includes resonance production and decay. Resonances can provide artificially enhanced radii because of their long lifetimes. Thus in hadron-hadron collisions $\rho$-mesons or $\Delta$-resonances may be produced with lifetimes of $1.3 \mathrm{fm} / \mathrm{c}$ or $1.7 \mathrm{fm} / \mathrm{c}$ respectively. Pions from the decay of these particles might well originate from outside the primary production zone, if it has a radius of $1 \mathrm{fm}$ or less, and lead to apparently larger radii.

In nucleus nucleus collisions with observed Bose-Einstein radii of 4-8 fm or more, $\rho-$ mesons and $\Delta$-resonances would provide only minor perturbations on the results. Of greater interest is the $\omega$-meson (lifetime $23 \mathrm{fm} / \mathrm{c}$ ). The Bose-Einstein correlation between pions from the decay of (uncorrelated) w-mesons would fall within a momentum difference of about i0 MeV/c, and would not have been observed in $\mathrm{e}^{+} \mathrm{e}^{-}$or hadron hadron experiments because of inadequate mimentum resolution. However observation of a radius of $8 \mathrm{fm}$ in NA35, implies that effects due to $\omega$ production might also ie observable. Such questions are under present study. While the results are exceedingly preliminary we show in Fig. 8 a spectrum from the reaction ${ }^{32} \mathrm{~S}+\mathrm{S}$ at 200 $\mathrm{GeV} /$ nucleon. It is interesting so sote a possible two-component structure in the correlation: a high, narrow, peak superimposed on a flat, broader one. Th arrow component corresponds to a radius of about $16 \mathrm{fm}$ and the wider component to a radius of abcut $4 \mathrm{fm}$. The latter radius is not unexpected, but the former is even larger than would be expected from the $\sqrt{\frac{d n}{d y}}$ dependence projected from the ${ }^{16} \mathrm{O}+\mathrm{Au}$ results. These results are at the moment no more than suggestive, but they emphasize the precision required in this kind of research, and the pitfalls that may arise in the interpretation. Information about $\omega$ production would be very interesting in its own right, 
but we need much further examination of the data as well as some model calculations of $\omega$ production and decay before we can draw any conclusions. Analysis of the results in terms of only one radius obviously would give a different result.

\subsection{Strange Particle Production}

An important test of quark gluon plasma formation is to measure the production of strange particles and to compare it with expectations for a hadronic gas [25]. The theoretically predicted yields depend on a variety of considerations. Lattice gauge calculations [26] predict an enhancement in an equilibrium situation. However, strangeness production cross sections in hadronic collisions are low, and it is not expected that equilibrium could be reached in a nucleus-nucleus collision if only hadronic interactions are involved. If a quark-gluon plasma is produced, several new processes involving gluons enhance the rate of strangeness production and enable it to approach the predicted equilibrium value during the limited time of the collision. These considerations have been discussed extensively in ref [27] and in a recent review [28].

The NA35 streamer chamber permits measurement of $\Lambda^{\circ}, \bar{\Lambda}^{\circ}$, and $K_{s}^{\circ}$ with an efficiency of the order of $10 \%$, via their $\mathrm{p} \pi^{-}, \overline{\mathrm{p}} \pi^{+}$and $\pi^{+} \pi^{-}$decays, and of $\mathrm{K}^{+}, \mathrm{K}^{-}$with much lower efficiency (of the order of $0.1 \%$ ) via their $3 \pi$ decay.

Results for central ${ }^{16} \mathrm{O}+$ Au collisions and for $\mathrm{p}+$ Au collisions at $60 \mathrm{GeV} /$ nucleon and 200 GeV/nucleon have been presented in ref [9]. A general picture of the production process emerges, but investigation of the question of quark gluon plasma production is hampered by inadequate predictions of production via hadronic processes. The standard model, FRITIOF [29] does not give an adequate description of strange baryon and antibaryon production in hadronic collisions, and furthermore does not explain the flow-widened $\mathrm{p}_{\mathrm{T}}$ distributions that are observed. Extraction of model independent quantities such as particle yield ratios is not possible because the experimental acceptances in $\left(y, p_{T}\right)$ are neither complete nor identical for different particles. 
However, the system ${ }^{32} \mathrm{~S}+\mathrm{S}$ is specially amenable to study and we present here some results at $200 \mathrm{GeV} /$ nucleon. For ${ }^{32} \mathrm{~S}+\mathrm{S}$ it is necessary to measure only the forward or the backward part of the rapidity distribution (because of the symmetry of the reaction) and we have been able to reconstruct a nearly complete picture of the total and differential yields and particle ratios, as well as to make comparisons with empirical nucleon-nucleon yields, with FRITIOF, and with other theoretical models.

Figures 9,10 show the yields of $\Lambda^{\circ}$ and $K_{s}{ }^{\circ}$ in ${ }^{32} \mathrm{~S}+\mathrm{S}$ collisions at $200 \mathrm{GeV} /$ nucleon, as a function of the $\pi^{-}$multiplicity. The three points plotted in each figure represent peripheral, intermediate , and central collisions respectively. The acceptances for $\Lambda^{\circ}$ were:

$$
\mathrm{p}>1.15 \mathrm{GeV} / \mathrm{c} ; 0.41 \mathrm{GeV} / \mathrm{c}<\mathrm{p}_{\mathrm{T}}<1.0 \mathrm{GeV} / \mathrm{c} ; 5.4^{\circ}<\theta<60^{\circ}
$$

and for $\mathrm{K}_{s}^{\circ}$

$$
\mathrm{p}>1.52 \mathrm{GeV} / \mathrm{c} ; 0.54 \mathrm{GeV} / \mathrm{c}<\mathrm{p}_{\mathrm{T}}<2.0 \mathrm{GeV} / \mathrm{c} ; 5.4^{\circ}<\theta<60^{\circ} ; \mathrm{y}<3 \text {. }
$$

The abscissa in figs 9,10 represents the number of non-strange hadrons observed in the streamer chamber acceptance:

$$
\mathrm{p}>0.1 \mathrm{GeV} / \mathrm{c} ; \theta<60^{\circ}
$$

and defined as

$$
<\mathrm{n}\rangle_{\mathrm{ns}}=\left\langle\mathrm{n}>-\left\langle\mathrm{n}_{\mathrm{K}}\right\rangle\right.
$$

where $\alpha \Omega$ is measured and $\left\langle\mathrm{n}_{\mathrm{K}^{-}}\right\rangle$is estimated using the measured $\mathrm{K}^{\circ}$ yield and the isospin relationship $\left\langle\mathrm{n}_{\mathrm{K}_{i}}\right\rangle=0.5\left(\left\langle\mathrm{n}_{\mathrm{K}^{+}}\right\rangle+\left\langle\mathrm{n}_{\mathrm{K}^{-}}\right\rangle\right)$for ${ }^{32} \mathrm{~S}+\mathrm{S}$, and measured values of the relative yields for $\mathrm{N}-\mathrm{N}$ collisions.

The $\Lambda^{\circ} / \pi$ and $\mathrm{K}_{\mathrm{s}}^{\circ} / \pi$ ratios shown in Figs 9,10 show a nearly linear increase with multiplicity; and reach 2-3 times the values expected from models consisting of a superposition of nucleon-nucleon collisions. 
Gazdzicki and Mrowczynski [30] have analyzed these results in terms of a partonic gas. Experimentally, the $y$ and $p_{T}$ distributions of $\pi^{-}, \Lambda^{\circ}$, and $K_{3}^{\circ}$ within their acceptances are approximately independent of the collision centrality, and the observed deviations from those for N-N collisions are small. The enhancement of $\Lambda^{\circ}$ and $K_{s}^{\circ}$ production by secondary interactions of pions with nucleons via the reaction $\pi+N \rightarrow \Lambda^{\circ}+K^{\circ}$ is not expected to be great, and this is confirmed by a similar enhancement (not shown) of $\bar{\Lambda}^{\circ}$ production, which could not be due to such processes. Thus the enhancements are ascribed to a closer approach to equilibrium in ${ }^{32} S+S$ central collisions than in $\mathrm{N}-\mathrm{N}$ collisions, resulting from the larger size of the interacting system. The degree of equilibration observed is hard to explain with hadronic collisions, but agrees quantitativeîy with estimates for a partonic gas.

The above results do not seem to depend significantly on the $\Lambda^{\circ}$ and $K_{s}^{\circ}$ acceptance of the experiment. Extrapolation to full phase space from our already large acceptance yields an erihancement of at least a factor of 2 for the relative strangeness concentration in ${ }^{32} \mathrm{~S}+\mathrm{S}$ compared with $\mathrm{N}-\mathrm{N}$ collisions.

\section{Discussion}

The experiments at CERN (and Brookhaven) have broken new ground in the characterization of ultrarelativistic nucleus-nucleus collisions. The NA35 experiment has provided a broad survey of the properties of these collisions, and has also provided suggestive evidence of new phenomena which may be related to quark-gluon-plasma formation.

The a priori case, presented in Sections 3.1 and 3.2, that the initial values of energy density and temperature reach those predicted to be necessary for quark-gluon-plasma formation, is strong. However, both the estimation of energy density and of temperature depend on untested assumptions about the collision dynamics. In Section 3.3 we presented evidence for transverse flow, using a model developed by Lee and Heinz [19] to articulate the collision dynamics. The width of the observed rapidity distribution [8] could be evidence for longitudinal flow. This is 
supported by analysis in terms of the Landau model $[31,32]$. However, the consequences of that model are not entirely clear. Since similar results are observed in p-p collisions at the same energies, the conclusion of ref. [32] that the results constitute evidence ícr a high degree of "stopping" should be regarded with caution.

A similar result would be obtained under the assumption of " complete transparency," if secondary particles are produced only in the volume of space-time when the projectile and target overlap. The uncertainty principle then guarantees momentum distributions which are wider longitudinally than transversely, reflecting the disk-like shape of the source.

In Section 4.1 we presented evidence of a large pion source at mid- rapidity. There is a great need for improved statistics, which is being accumulated. Once again, the interpretation depends on an understanding of the expansion dynamics, but it is hard to see how a source which is coalescing into pions at a radius of $8 \mathrm{fm}$ could be explained at its original radius more conveniently as a hadronic gas than in terms of the dynamics of partons. Even if a partonic description is more appropriate, this does not necessarily imply a quark-gluon plasma in an equilibirum. state. Much more data should soon be forthcoming, but the accuracy of the measurements will enforce a complete understanding of the role of resonance production, if any.

In Section 4.2 evidence was presented for an enhancement of $\Lambda^{\circ}, \bar{\Lambda}^{\circ}$, and $K_{s}^{\circ}$ production in ${ }^{32} \mathrm{~S}+\mathrm{S}$ central collisions. The evidence here is becoming quite solid, independent of assumptions. The interpretation in terms of a partonic gas seems to be rather insensitive to assumptions about energy densities and temperatures, the principal variable being the size of the interaction volume. The symmetry of the ${ }^{32} \mathrm{~S}+\mathrm{S}$ system enabled a more complete investigation of strangeness producion than was possible in $\mathrm{p}+\mathrm{Au}$ or $\mathrm{O}+$ Au collisions, where central collisions are also complicated by the presence of spectator matter. Correlation between the two sets of data depends on a number of assumptions and on the use of models which are not yet reliable enough. However, the ${ }^{32} \mathrm{~S}+\mathrm{S}$ results reach a natural interpretation in terms of a quark-gluon plasma, and 
should be interesting for future theoretical study. It should also be interesting to study these results in the context of existing data and analysis for hadron-hadron collisions. Here strangeness production is usually discussed in terms of a "strangeness suppression factor" $\lambda_{s}$, defined as the

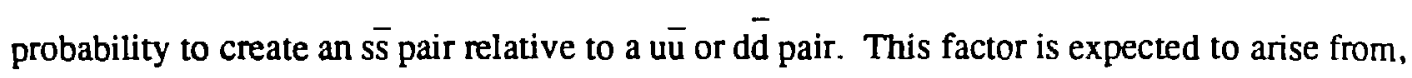
for example, the difference in masses of $u, d$, and s quarks. Recently Geist [33] has reviewed the data on $\lambda_{3}$ for kaon production in $\mathrm{e}^{+} \mathrm{e}^{-}$annihilation, deep inelastic lepton-hadron, and hadronhadron collisions. The value of $\lambda_{\mathbf{s}}$ expected depends on the reaction and on the kinematic domain within a given reaction. The value of $\lambda_{\mathrm{s}}$ is as large as 0.5 under some conditions. A combined investigation of strangeness enhancement (and suppression) in nucleus-nucleus collisions and in the more microscopic collisions may be in order.

\section{Future Plans}

The experiments carried out so far are only a beginning. Further runs in 1990 and 1991 are planned at the SPS, and most of the experiments will have improved equipment. The NA35 Collaboration plans to add [35] ring imaging Cherenkov (RICH) detectors to improve particle identification near mid-rapidity, and a Time Projection Chamber (TPC) in the iorward direction to track particles which were previously lost because of the high density of unresolved tracks in the streamer chamber. The TPC will also allow tests of particle identification using $\mathrm{dE} / \mathrm{dx}$ measurement in the relativistic rise regime, and provide information on $\mathrm{p}, \overline{\mathrm{p}}, \mathrm{K}^{ \pm}$and $\pi^{\ddagger}$ yields in the forward direction. The layout for the revised experiment is shown in Figure 11.

CERN has been studying the possibility of extending the SPS program to much heavier projectiles such as $\mathrm{Pb}$ at $170 \mathrm{GeV} /$ nucleon [36]. At present it seems possible that these might become available in 1993. In anticipation of this, NA35 has proposed to join forces with WA80 to build a large hadron and photon detector with almost complete coverage and particle identification forward of mid-rapidity for $\mathrm{Pb}+\mathrm{Pb}$ collisions. Because of the collision symmetry, most of the reaction dynamics should be accessible to such a detector. The tentative layout of the 
detector is shown in Figure 12. Neither the NA35 streamer chamber or the WA80 Plastic Ball is of use in the increased multiplicity environment (of the order of 2000 charged and 2000 neutral parcicles per central collision) and they are abandoned in favor of a large TPC. This TPC, with $100.200,000$ channels, when combined with $12 \mathrm{~m}^{2}$ of RICH detectors, would track and identify 1000 charged particles. A Bismuth Germanate (BGO) photon detector with 3200 modules is added to complement the TPC by measurement of $\pi^{\circ}, \eta^{\circ}$, and also to look for direct photon production, which should be enhanced $[38,39]$ if a quark-gluon plasma is produced.

Experiments with $\mathrm{Pb}$ beams will present a difficult challenge to the experimenters. The challenge already presented to theorists to understand the already existing results with ${ }^{16} \mathrm{O}$ and ${ }^{32} \mathrm{~S}$ beams will be intensified, or perhaps reduced by the observation of qualitatively new phenomena characteristic of large nuclear systems as opposed to the present results which may be more closely related to smaller systems such as p-p or p-nucleus.

\section{Acknowledgements}

This work was supported by the Director, Office of Energy Research, Division of Nuclear Physics of the Office of High Energy and Nuclear Physics of the U.S. Department of Energy, under contract No. DE-AC03-76SF00098. 


\section{References}

[1] e. g. J.B. Kogut, Nucl. Phys. A4l8a, 381c (1984) H. Satz, Nucl. Phys. A418, 447c (1984)

[2] A Sandoval et al., Nucl. Phys. $A 461,465 \mathrm{c}\left(198{ }^{\circ}\right.$ )

[3] M. L Tincknell et al., Opt. Eng. 26, 1067 (1987).

[4] A. Bamberger et al., (NA35 Collaboration), Phys. Lett. B184, 271 (1987); W. Heck et al.. (NA35 Collaboration), 2. Phys. C38, 19 (1988).

[5] A. Bamberger et al., (NA35 Collaboration), Phys. Lett. B205, 583 (1988)

[6] A. Bamberger et ail., (NA35 Collaboration), Phys. Lett. B203, 320 (1988).

[7] T. J. Humanic et al., (NA35 Collaboration), Z. Phys. C38, 79 (1988).

[8] H. Ströbule et al. (NA35 Collaboration), Z. Phys. C38, 89 (1988).

[9] A. Bamberger et al., (NA35 Collaboration), Phys. Z. Phys. C43, 25 (1989).

[10] M. Gazdzicki et al., (NA35 Collaboration), Nucl. Phys. A498 375c (1989); R. Renfordt et al., (NA35 Collaboration), Nucl. Phys. A498 385c (1989);

[11] H.G. Pugh, Acta Physica Polonica B19, 307 (1988).

[12] R. Stock, Nucl. Phys. A488, 599c (1988).

[13] J.W. Harris, Nucl. Phys. A498, 133c (1989).

[14] General references on this topic may be found in the proceedings of the various "Quark Matter" conference proceedings, most recently: Quark Matter 1987, Nordkirchen, 24-28 August, 1987, ed. H. Satz, H.J. Specht, R. Stock, Z. Phys. C38 (1988); and Quark Matter 1988, Lenox, 26-30, September, 1988, ed. G. Baym, P. Brown-Munzinger and S. Nagamiya, Nucl. Phys. A498 (1989).

[15] J.D. Bjorken, Phys Rev. D27, 140 (1983). 
[16] R. Hagedom. CERN 71-12 (1971); R. Hagedom, Proceedings of the Workshop on Future Relativistic Heavy Ion Experiments, GSI Darmstadt, Oct. 7-10, 1980, GSI 81-6 (1981) 236: R. Hagedom, Quark Matter 84, ed. K. Kajantie, Springer-Verlag (1985) 53.

[17] T. Abbott et al., (E802 Collaboration) Nucl. Phys. A498, 67c (1989).

[18] P.J. Siemens and J.O. Rasmussen, Phys. Rev. Lett. 42, 880 (1979).

[19] K.S. Lee and U. Heinz, Z. Phys. C43, 425 (1989).

[20] J.Y. Grossiord et al., (NA35 Collaboration), Nucl. Phys. A498 249c (1989);

[21] T. Matsui and H. Satz, Phys. Rev. Lett. $178 B, 416$ (1986).

[22] M. Gyulassy and S.S. Padula, Phys. Rev. Lett. 217B, 181 (1986).

[23] G. Bertsch, M. Gong, and M. Tohyama, Phys. Rev. C37, 1896 (1988); and G. Bertsch, Nucl. Phys A498, 73c (1989).

[24] K. Kajantie, Nucl. Phys A498, 355c (1989).

[25] R. Hagâfom and J. Rafelski, Phys. Rev. Lett. 97B, 180 (1980).

[26] J.B. Kogut and D.K. Sinclair, Phys. Rev. Lett. 60, 1250 (1980).

[27] P. Koch, B. Müller, and J. Rafelski, Phys. Rep. 142, 453 (1986).

[28] B. Müller Invited talk at "Hadronic Matter in Collision," Tucson, October 1988, to be published by World Scientific, Univ. of Frankfurt preprint UFTP 227/1989.

[29] B. Andersson et al., Nucl. Phys. B281, 289 (1987).

[30] M. Gazdzicki and S. Mrowczynki, Univ. of Regensburg Reprint TPR-89-7 (1989).

[31] H. von Gersdorff et al., Phys. Rev. C39, 1385 (1989).

[32] J. Stachel and P. Braun-Munzinjer, Phys. Lett. B216, 1 (1989).

[33] W. M. Geist, Phys. Lett. B196, 251 (1987). 
[34] H. G. Fischer, Z. Phys. C38, 105 (1988).

[35] CERN-SPSC-89-17, SPSC/M-440, March 6, 1989.

[36] P. Darriulat, Z. Phys. C38, 325 (1988).

[37] CERN/SPSC 89-73, SPSC/I-173, July 4, 1989.

[38] E. V. Shuryak, Phys. Lett. B78, 150 (1978).

[39] R. Santo, Nuclear Physics A498, 391c (1989). 
Figure Captions

Fig. 1. The NA35 experimental set up.

Fig. 2. Streamer chamber picture of a central ${ }^{16} \mathrm{O}+\mathrm{Pb}$ collision at $200 \mathrm{GeV} /$ nucleon, together with the angular acceptance of the mid-rapidity calorimeters (PPD and Ring) and the leading fragment calorimeter (Veto).

Fig. 3. Mid-rapidity transverse energy distribution for ${ }^{16} \mathrm{O}+\mathrm{Pb}$ at $200 \mathrm{GeV} /$ nucleon. The solid line is a 16-fold convolution of the distribution for inelastic $\mathrm{p}+\mathrm{Au}$ collisions at $200 \mathrm{GeV}$, measured with the same apparatus.

Fig. 4. Transverse mass distributions of various particles in central ${ }^{32} \mathrm{~S}+\mathrm{S}$ collisions at 200 $\mathrm{GeV} / \mathrm{A}$. The rapidity intervals for $\Lambda^{\circ}, \mathrm{p}, \mathrm{K}_{\mathrm{s}}^{\circ}, \pi^{-}$are $0.8<\mathrm{y}<2.0,1.5<\mathrm{y}<3.0$, $1.4<y<2.7,1.5<y<3.5$, respectively. The lines are to indicate the slope corresponding to a temperature of $200 \mathrm{MeV}$.

Fig. 5. The same as for Fig. 4, but for ${ }^{16} \mathrm{O}+$ Au collisions at $200 \mathrm{GeV} / \mathrm{A}$. Acceptances are indicated.

Fig. 6. The same as for Fig. 4, but for $\mathrm{p}+\mathrm{Au}$ collisions at $200 \mathrm{GeV} / \mathrm{A}$. Acceptances are indicated.

Fig. 7. Transverse momentum distributions for central ${ }^{16} \mathrm{O}+\mathrm{Au}$ collisions at $200 \mathrm{GeV} / \mathrm{A}$ for (a) negatively charged particles at $2<y<3$;

(b) $\mathrm{K}_{\text {; }}^{\circ}$ at $1.7<\mathrm{y}<2.8$;

(c) $\Lambda^{\circ}$ at $1.5<y<2.4$;

(d) $\bar{\Lambda}^{\circ}$ at $1.5<y<2.4$.

Also shown are predictions of the radial expansion mudei of Lee and Heinz [19] with parameters adjusted to fit the spectrum of negatives, as described in the text. 
Fig. 8. Correlation function (q-invariant) for central ${ }^{32} \mathrm{~S}+\mathrm{S}$ collisions at $200 \mathrm{GeV} / \mathrm{A}$ in the rapidity interval $1.5<y<2.5$. These results are in a very preliminary stage of analysis and are shown only to illustrate the possibilities which have to be investigated as the analysis proceeds.

Fig. 9. Rates of the mean multiplicity of $\Lambda^{\circ}$ in the acceptance $A 1$ (defined in the text) to the multiplicity of non-strange negative hadrons as a function of the latter. Closed circles correspond to ${ }^{32} \mathrm{~S}-\mathrm{S}$ collisions at $200 \mathrm{GeV} / \mathrm{A}$, whereas the open one is for nucleon-nucleon interactions.

Fig. 10. The same as for Fig. 9, but for $\mathrm{K}_{\mathrm{s}}^{\circ}$ in the $\mathrm{A} 2$ acceptance.

Fig. 11. Layout of the 1990-91 NA35 experiment, showing the location of the NA35 TPC in the forward direction, and the new RICH detectors.

Fig. 12. Tentative layout of the large hadron and photon detector presented by NA35 and WA80 in their Letter of Intent to the CFRN SPSC, for the anticipated program using $\mathrm{Pb}$ beams at $170 \mathrm{GeV} / \mathrm{A}$. 


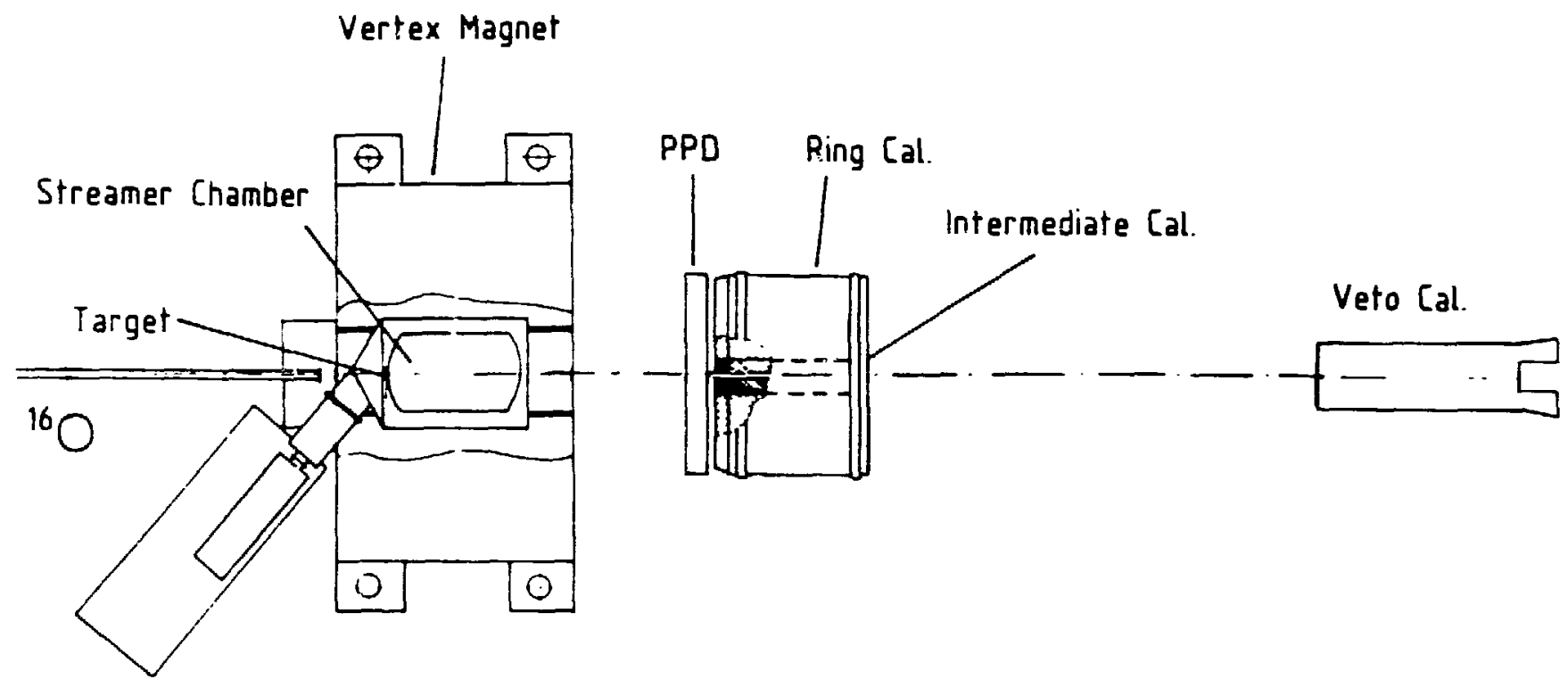

Fig. 1. The NA35 experimental set up. 


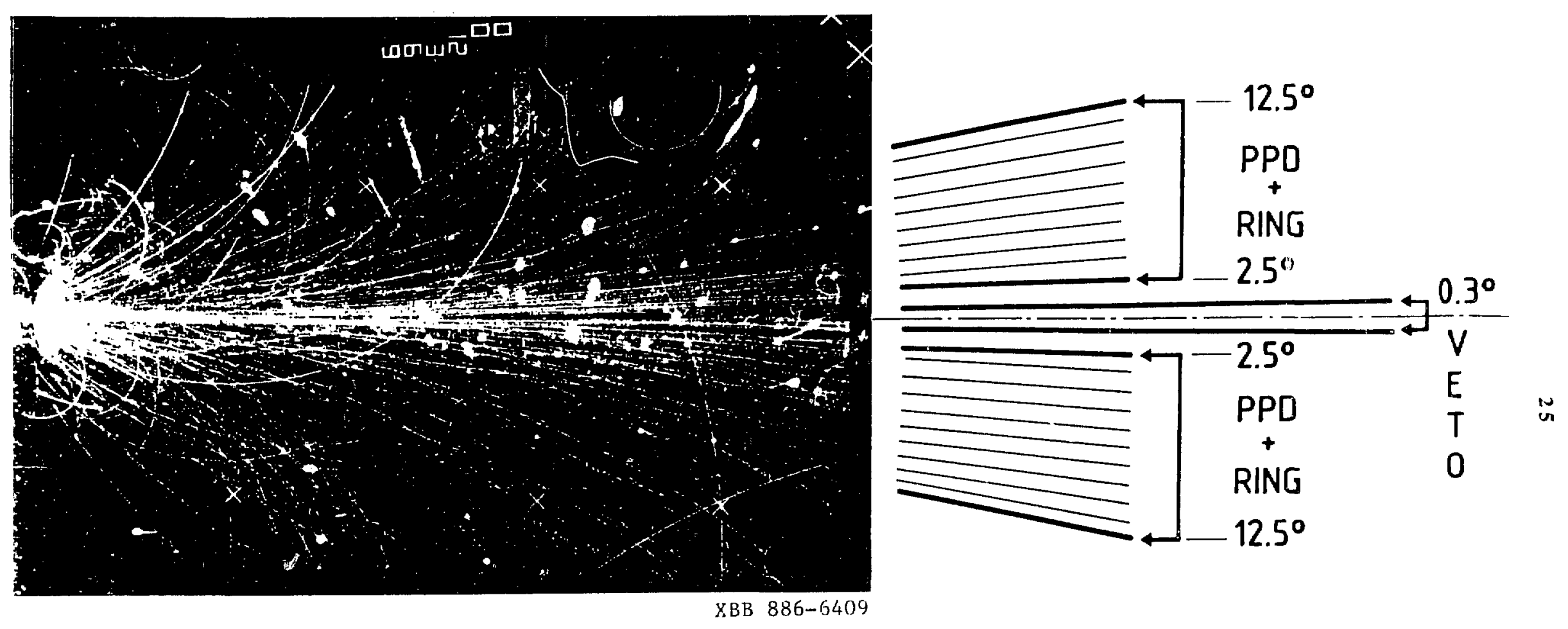

Fig. 2. Streamer chamber picture of a central ${ }^{16} \mathrm{O}+\mathrm{Pb}$ collision at $200 \mathrm{GeV}$ /nucleon, together with the angular acceptance of the mid-rapidity calorimeters (PPD and Ring) and the leading fragment calorimeter (Veto). 


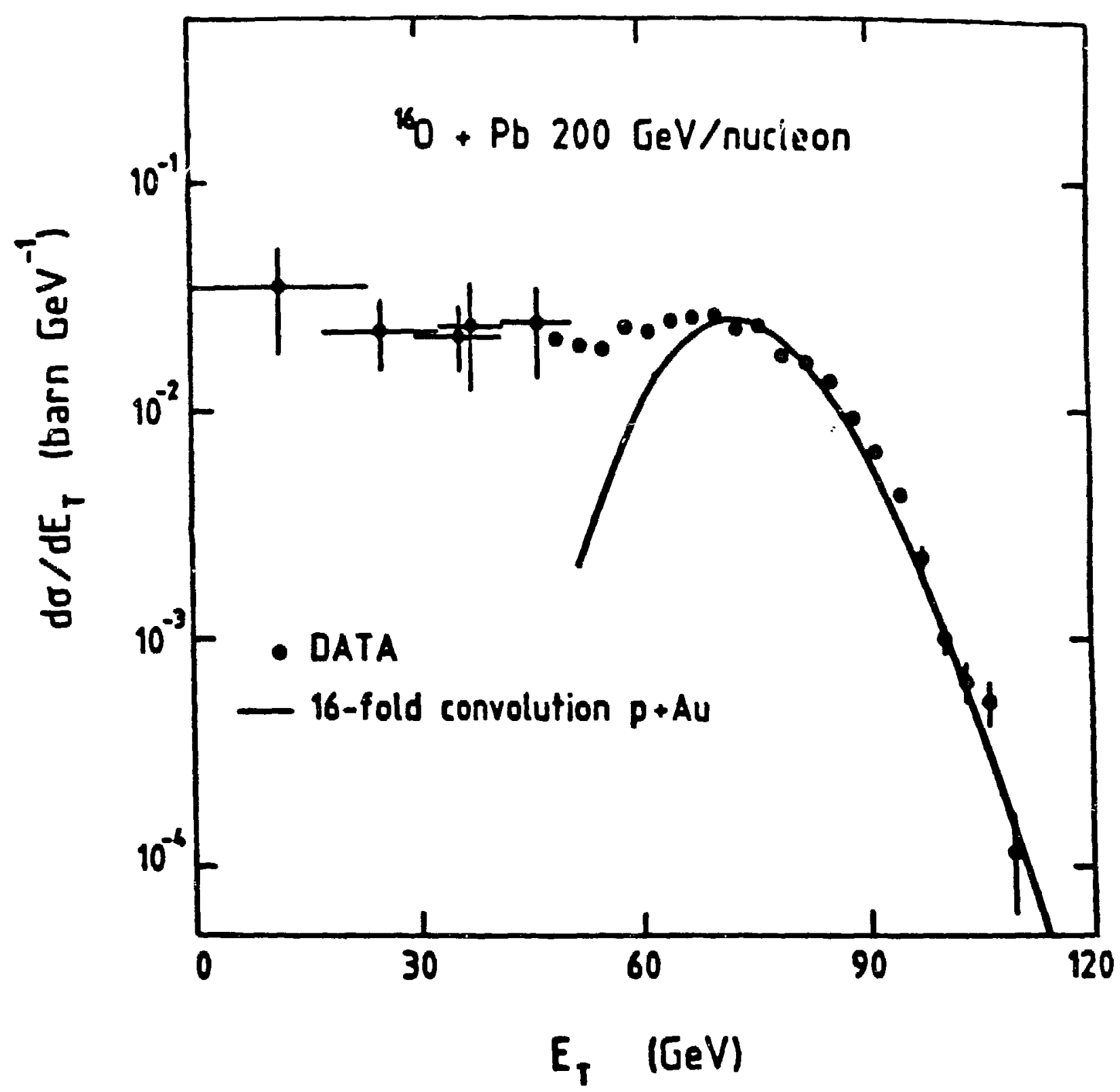

Fig. 3. Mid-rapidity transverse energy distribution for ${ }^{16} \mathrm{O}+\mathrm{Pb}$ at $200 \mathrm{GeV} /$ nucleon. The solid line is a 16 -fold convolution of the distribution for inelastic $p+$ Au collisions at $200 \mathrm{GeV}$, measured with the same apparatus. 


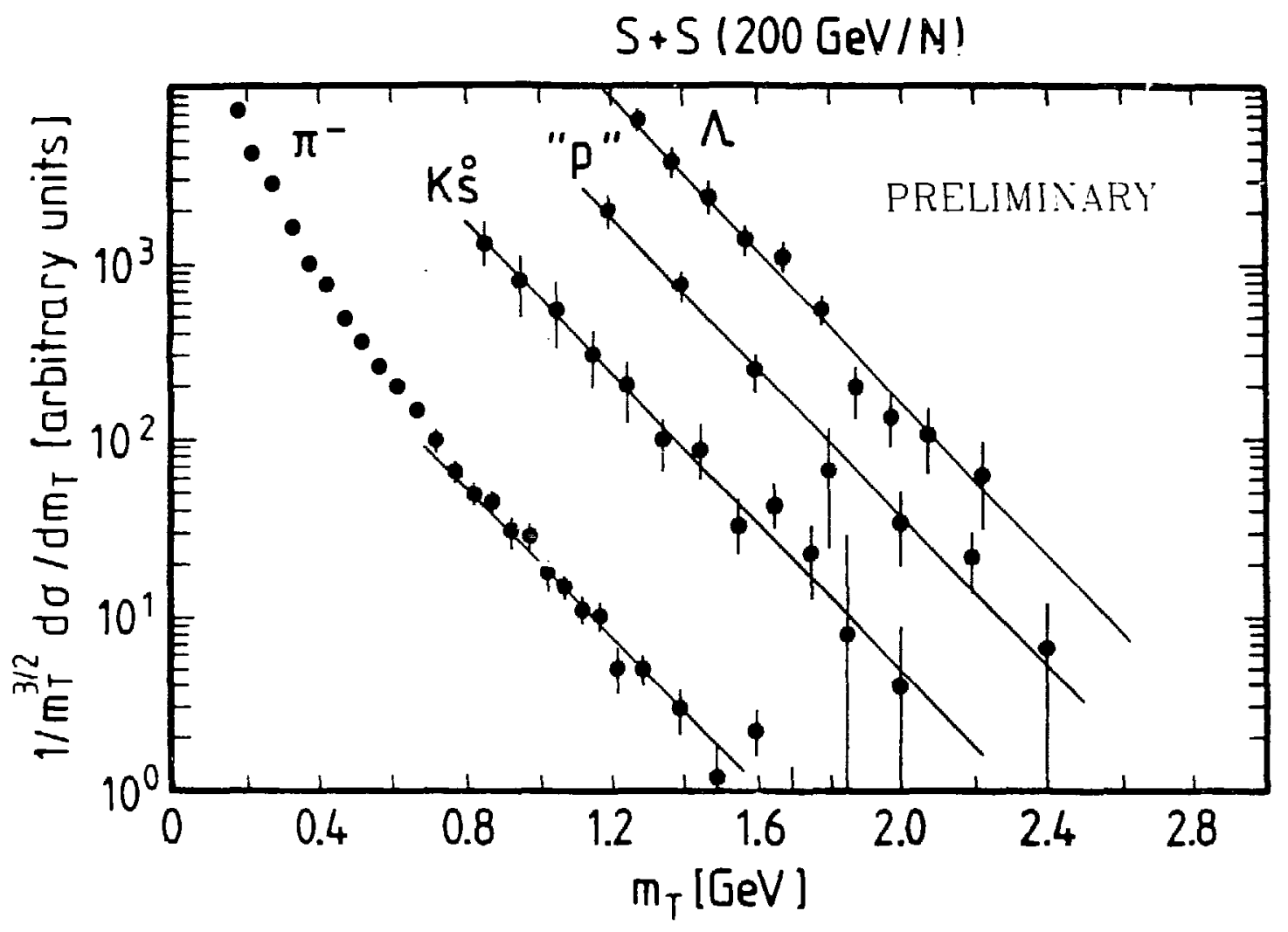

Fig. 4. Transverse mass distributions of various particles in central ${ }^{32} \mathrm{~S}+\mathrm{S}$ collisions at 200 $\mathrm{GeV} / \mathrm{A}$. The rapidity intervals for $\Lambda^{\circ}, \mathrm{p}, \mathrm{K}_{\mathrm{s}}^{0}, \pi^{-}$are $0.8<\mathrm{y}<2.0,1.5<\mathrm{y}<3.0$, $1.4<y<2.7,1.5<y<3.5$, respectively. The lines are to indicate the slope corresponding to a temperature of $200 \mathrm{MeV}$. 


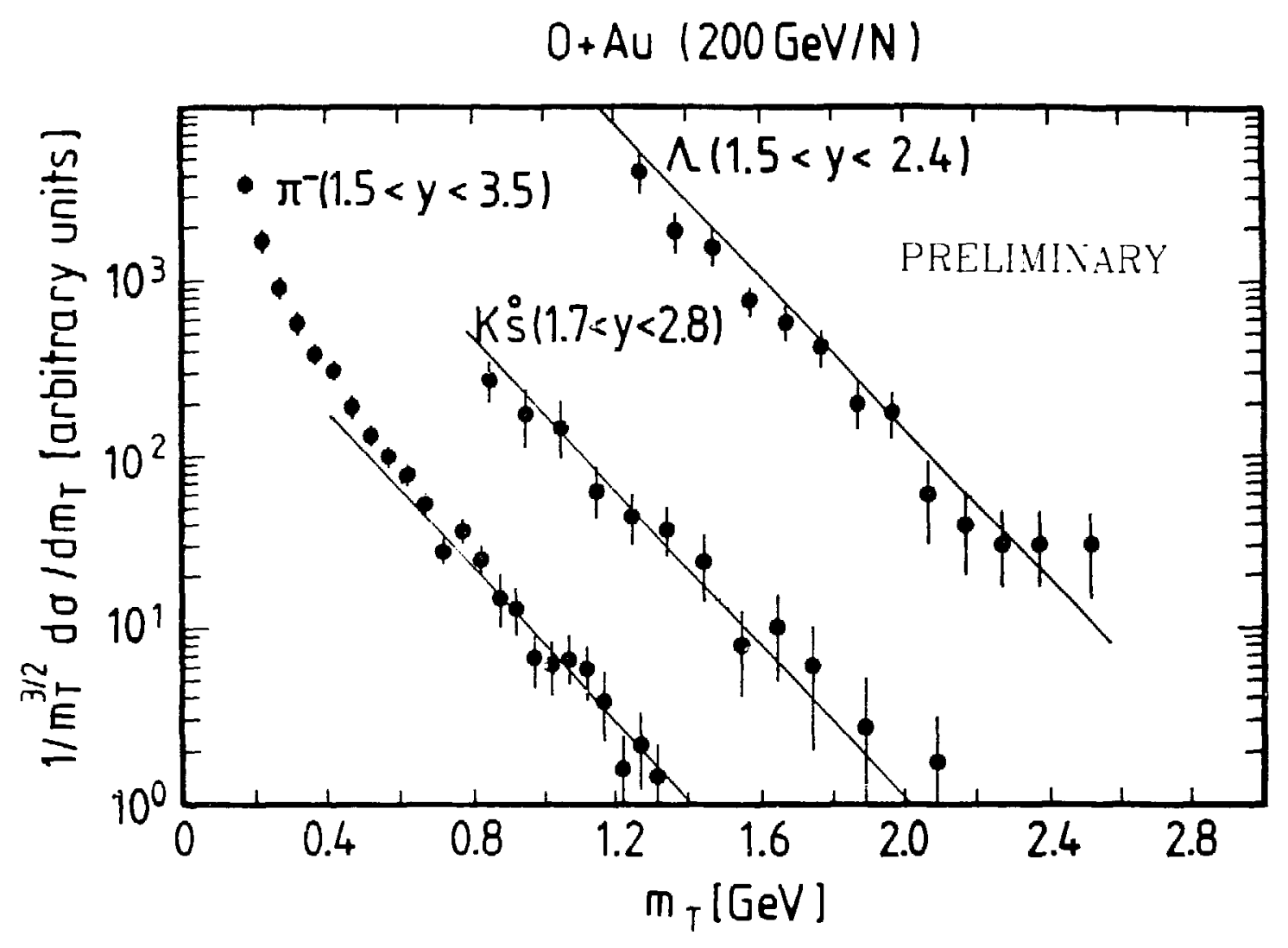

Fig. 5. The same as for Fig. 4, but for ${ }^{16} \mathrm{O}+$ Au collisions at $200 \mathrm{GeV} / \mathrm{A}$. Acceptances are indicated. 


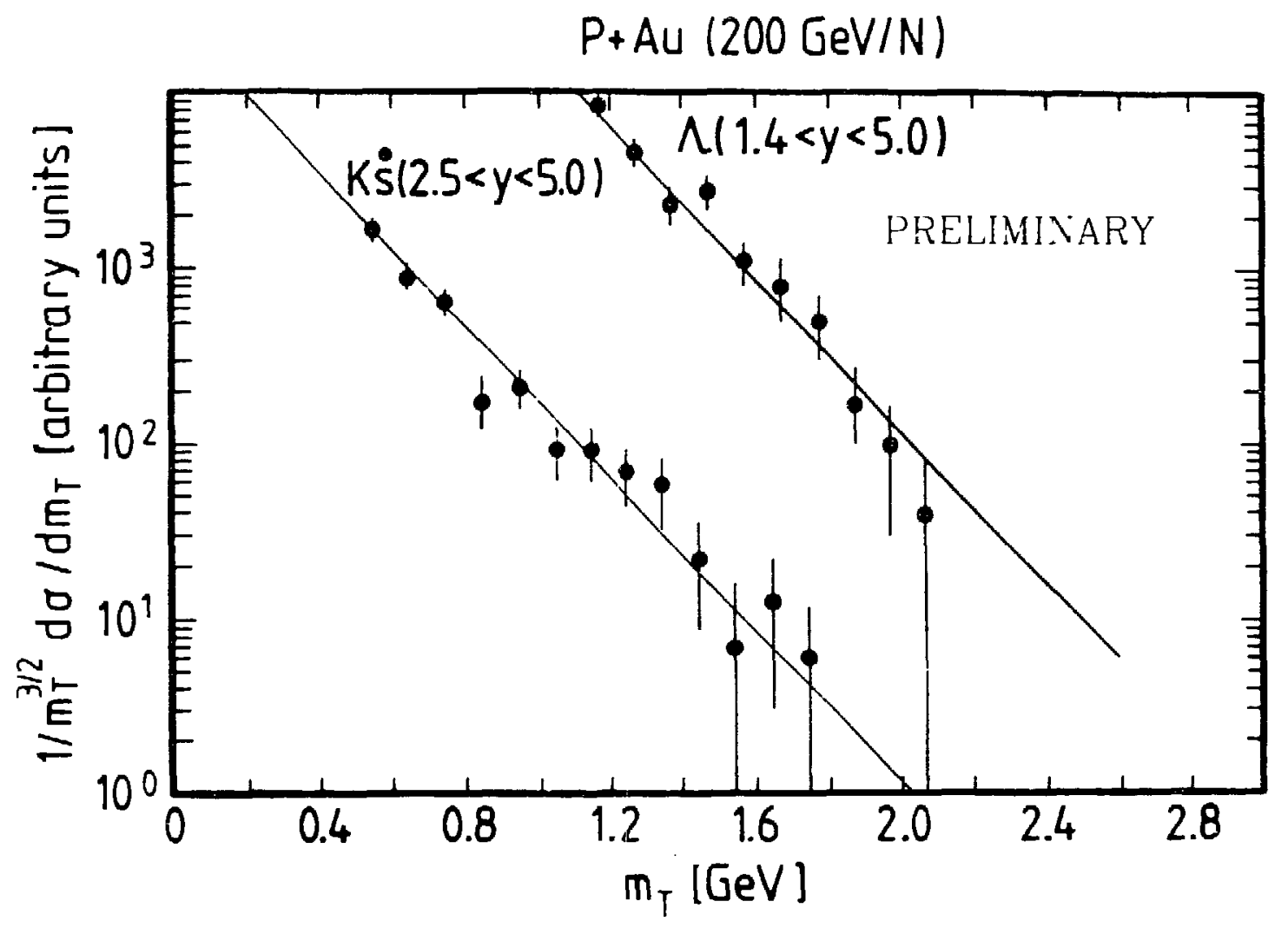

Fig. 6. The same as for Fig. 4. but for $\mathrm{p}+\mathrm{Au}$ collisions at $200 \mathrm{GeV} / \mathrm{A}$. Acceptances are indicated. 

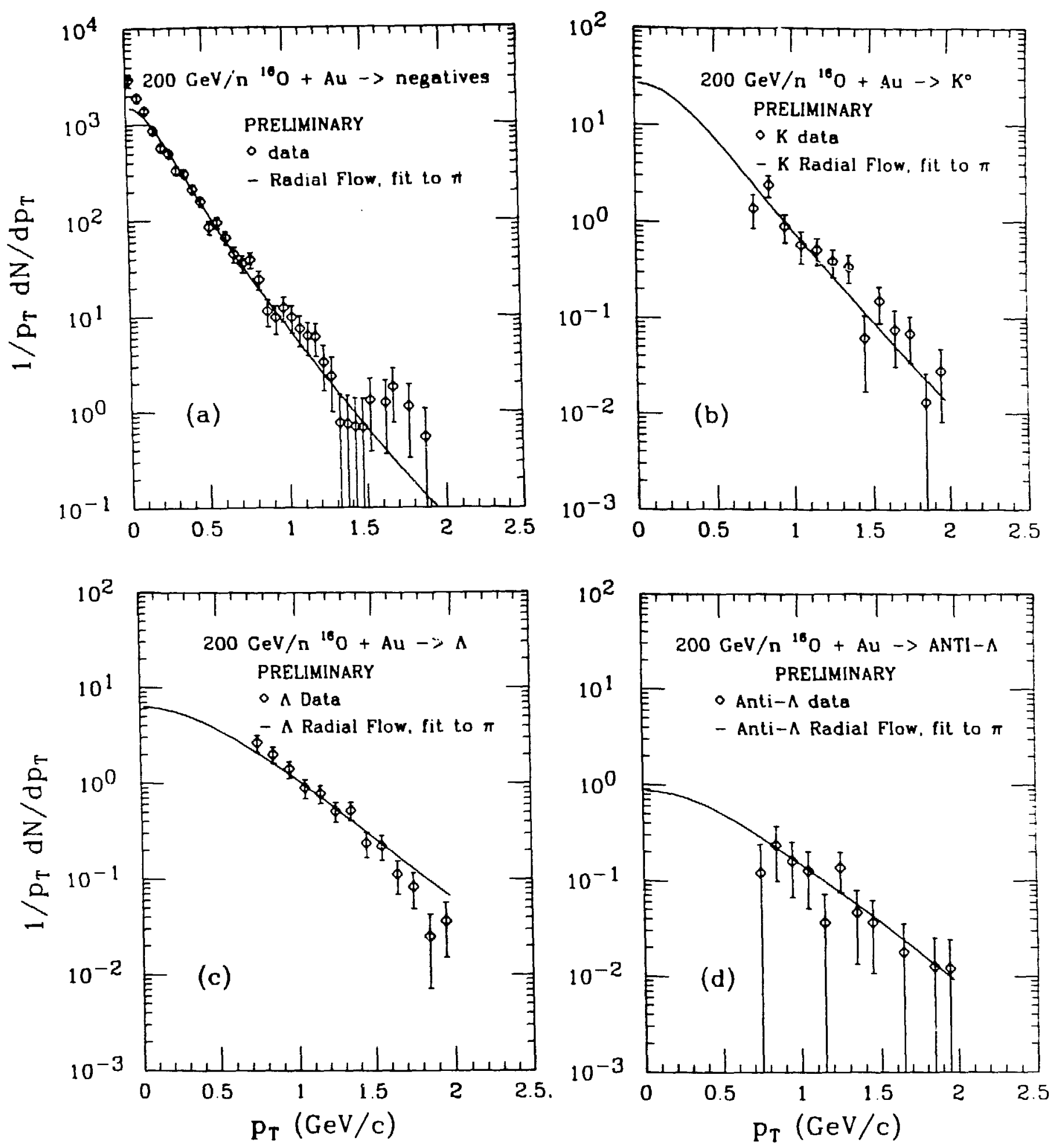

Fig. 7. Transverse momentum distributions for central ${ }^{16} \mathrm{O}+\mathrm{Au}$ collisions at $200 \mathrm{GeV} / \mathrm{A}$ for

(a) negatively charged particles at $2<y<3$;

(b) $\mathrm{K}_{3}$ at $1.7<\mathrm{y}<2.8$;

(c) $\Delta^{\circ}$ at $1.5<y<2.4$;

(d) $\Lambda^{\circ}$ at $1.5<y<2.4$.

Also shown are predictions of the radial expansion model of Lee and Heinz [19] with parameters adjusted to fit the spectrum of negatives, as described in the text. 


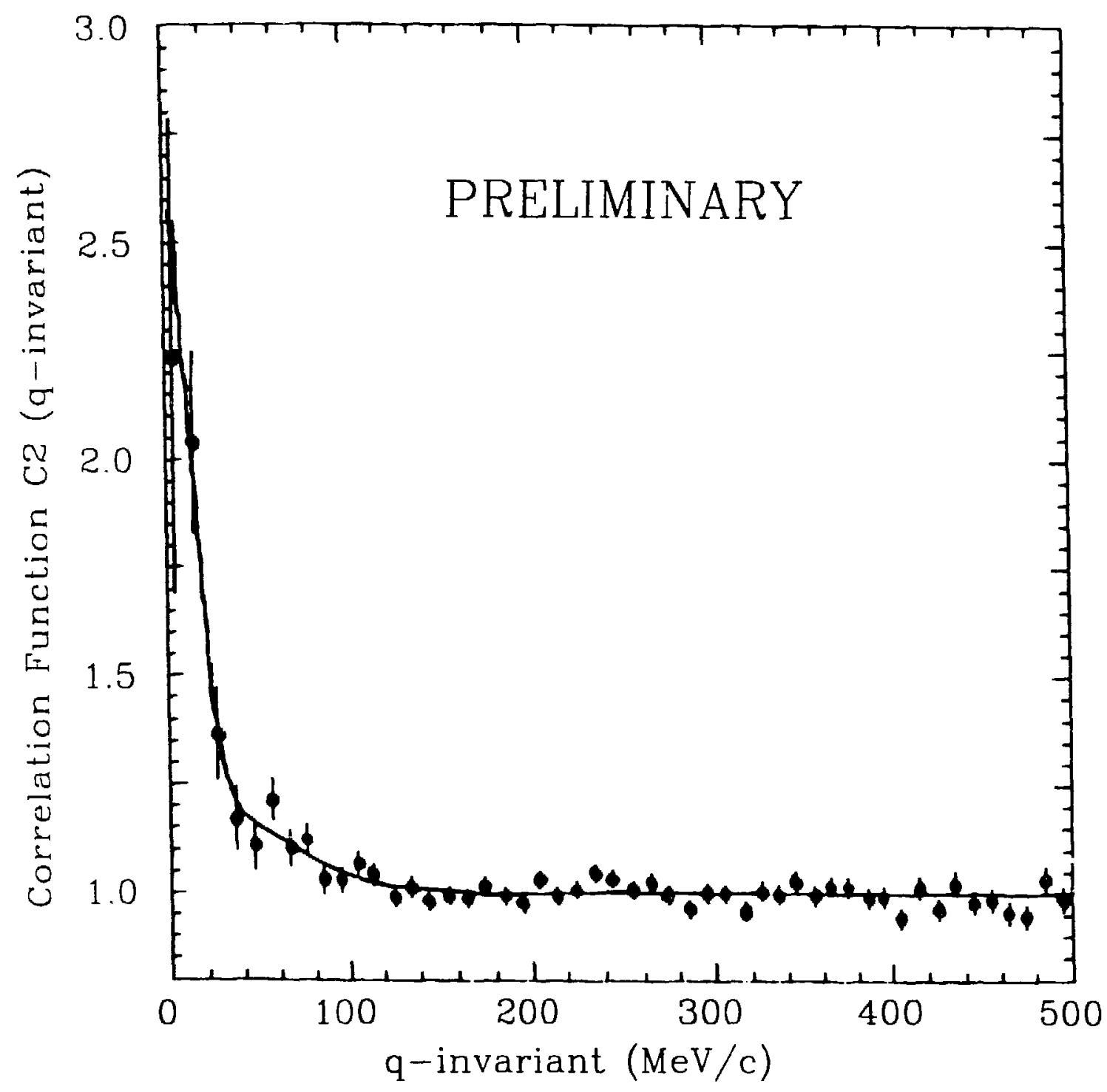

Fig. 8. Correlation function ( $q$-invariant) for central ${ }^{32} \mathrm{~S}+\mathrm{S}$ collisions at $200 \mathrm{GeV} / \mathrm{A}$ in the rapidity interval $1.5<y<2.5$. These results are in a very preliminary stage of analysis and are shown only to illustrate the possibilities which have to be investigated as the analysis proceeds. 


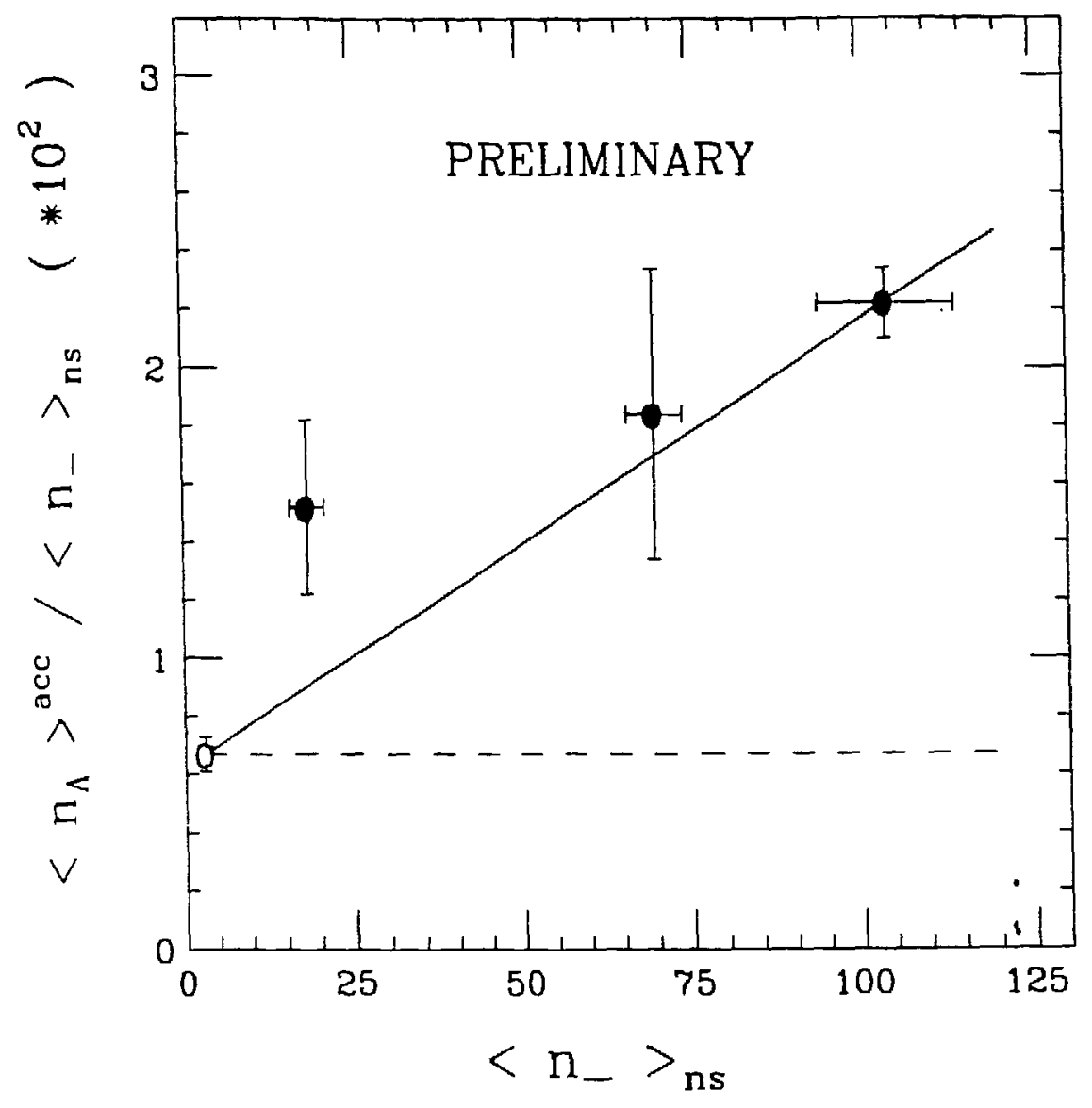

Fig. 9. Rates of the mean multiplicity of $\Lambda^{\circ}$ in the acceptance $A 1$ (defined in the text) to the multiplicity of non-strange negative hadrons as a function of the latter. Closed circles correspond to ${ }^{32} \mathrm{~S}-\mathrm{S}$ collisions at $200 \mathrm{GeV} / \mathrm{A}$, whereas the open one is for $\mathrm{N}-\mathrm{N}$ interactions. 


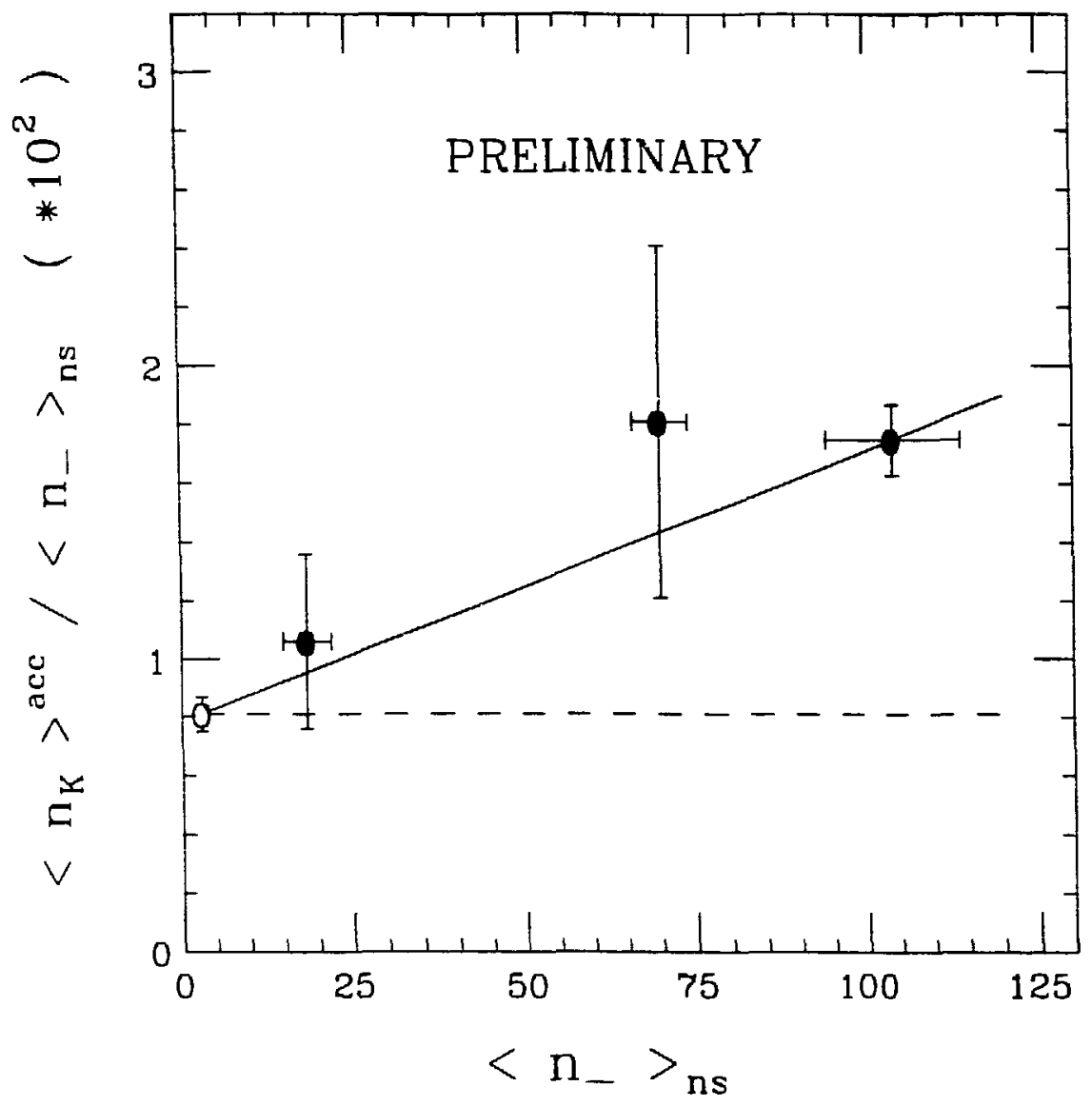

Fig. 10. The same as for Fig. 9, but for $\mathrm{K}_{\mathrm{s}}{ }^{\circ}$ in the $\mathrm{A} 2$ acceptance. 


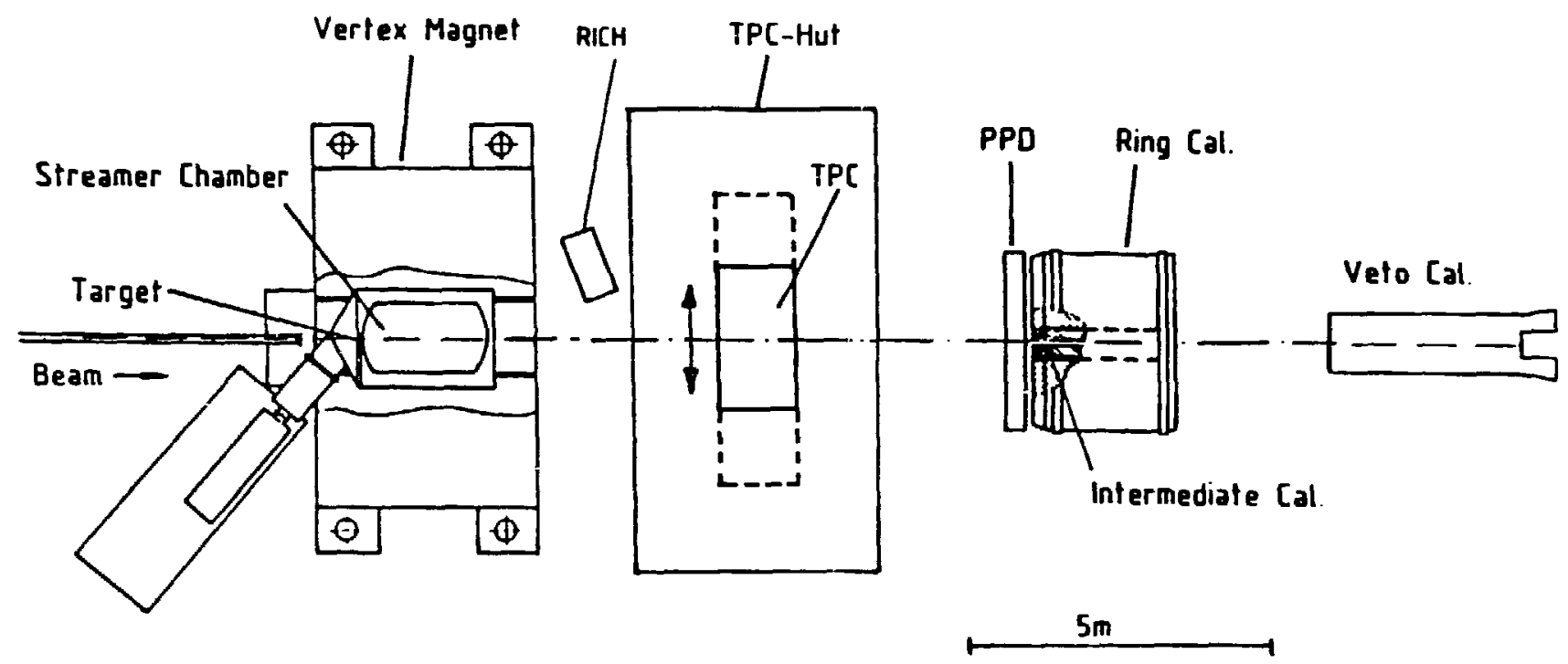

Fig. 11. Layout of the 1990-91 NA35 experiment, showing the location of the NA35 TPC in the forward direction, and the new RICH detectors. 


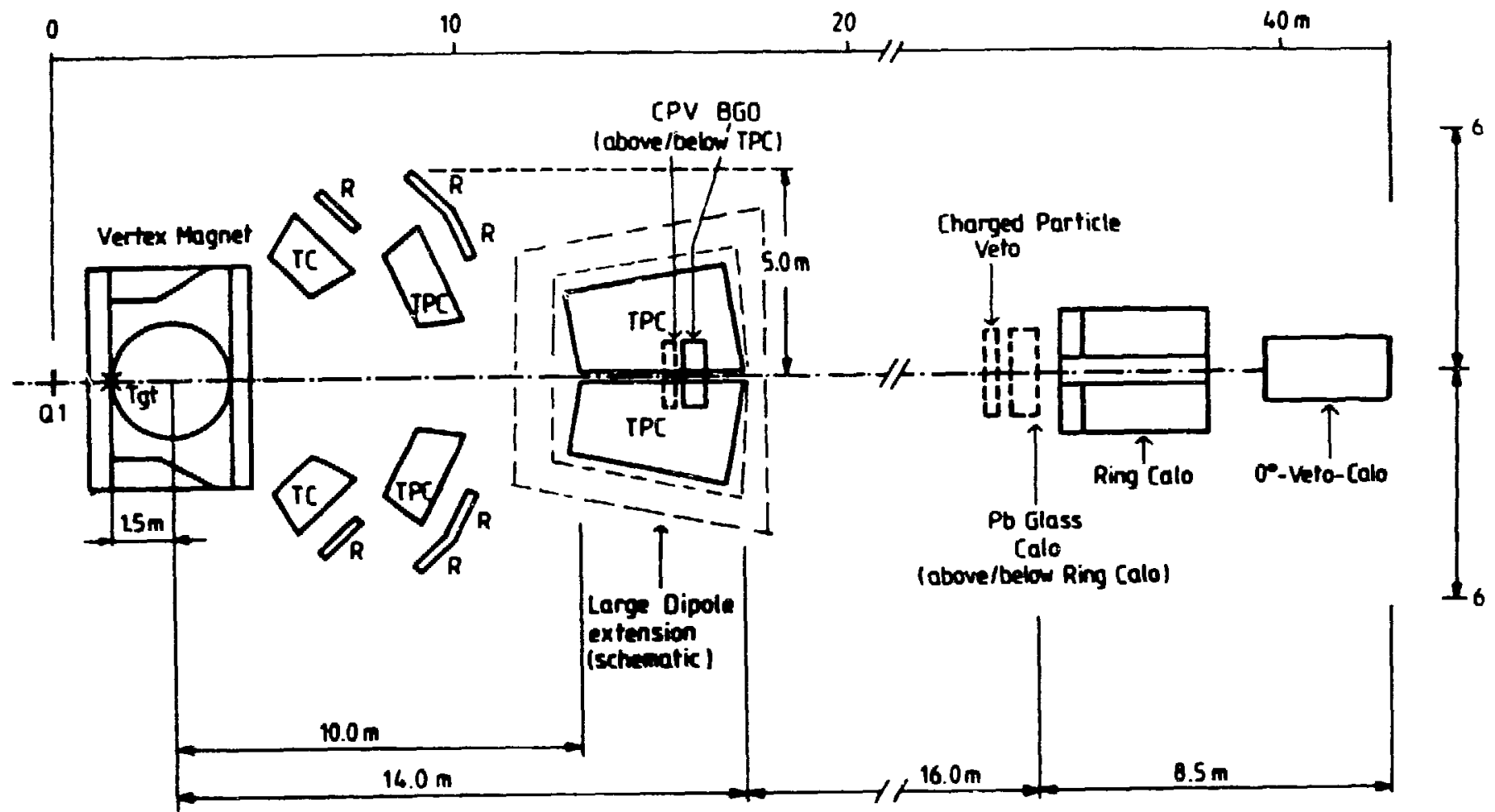

Fig. 12. Tentative layout of the large hadron and photon detector presented by NA35 and WA80 in their Letter of Intent to the CERN SPSC, for the anticipated program using $\mathrm{Pb}$ beams at $170 \mathrm{GeV} / \mathrm{A}$.

\section{DISCLAIMER}

This report was prepared as an account of work spensored by an agency of the United States Government. Neither the United States Government nor any agency thereof, nor any of their employees, makes any warranty, express or implied, or assumes any legal liability or responsibility for the accuracy, completeness, or usefulness of any information, apparatus, product, or process disclosed, or represents that its use would not infringe privately owned rights. Reference herein to any specific commercial product, process, or service by trade name, trademark, manufacturer, or otherwise does not necessarily constitute or imply its endorsement, recomniendation, or favoring by the United States Government or any agency thereof. The views and epinions of authors expressed herein do not necessarily state or reflect those of the United States Government or any agency thereof. 